\title{
Development of a nomogram for predicting the operative mortality of patients who underwent pneumonectomy for lung cancer: a population-based analysis
}

\author{
Xiangyang Yu\#, Shugeng Gao", Qi Xue, Fengwei Tan, Yushun Gao, Yousheng Mao, Dali Wang, Jun Zhao, \\ Yin Li, Feng Wang, Hong Cheng, Chenguang Zhao, Juwei Mu \\ Department of Thoracic Surgery, National Cancer Center/National Clinical Research Center for Cancer/Cancer Hospital, Chinese Academy of \\ Medical Sciences and Peking Union Medical College, Beijing, China \\ Contributions: (I) Conception and design: X Yu, F Tan, Y Mao, J Mu; (II) Administrative support: S Gao, Q Xue, D Wang, J Zhao, J Mu; (III) \\ Provision of study materials or patients: X Yu, F Wang, H Cheng, C Zhao; (IV) Collection and assembly of data: X Yu, Y Gao, Y Mao, D Wang, J \\ Zhao, Y Li; (V) Data analysis and interpretation: X Yu, J Zhao, Y Li; (VI) Manuscript writing: All authors; (VII) Final approval of manuscript: All \\ authors. \\ "These authors contributed equally to this work. \\ Correspondence to: Prof. Juwei Mu. Department of Thoracic Surgery, National Cancer Center/National Clinical Research Center for Cancer/Cancer \\ Hospital, Chinese Academy of Medical Sciences and Peking Union Medical College, Beijing, China. Email: mujuwei@cicams.ac.cn.
}

Background Although many studies have reported that patients have undergone entire lung removal for lung cancer along with high operative mortality, the trends in the incidence and associated risk factors for operative death have not been explored in a national population-based study. In addition, a clinical decisionmaking nomogram for predicting postpneumonectomy mortality remains lacking.

Methods: A total of 10,337 patients diagnosed with lung cancer who underwent pneumonectomy between 1998 and 2016 were retrieved from the Surveillance, Epidemiology, and End Results (SEER) cancer registry. Multivariate logistic regression analysis was used to identify risk factors for predicting operative mortality. Thereafter, these independent predictors were integrated into a nomogram, and bootstrap validation was applied to assess the discrimination and calibration. Additionally, decision curve analysis (DCA) was used to calculate the net benefit of this forecast model.

Results: The overall postpneumonectomy mortality between 1998 and 2016 was $10.3 \%$, including a 30-day mortality of $4.2 \%$; however, there were statistically significant decreases in the operative death rates from $8.8 \%$ in 1998 to $6.7 \%$ in 2016 ( $\mathrm{P}=0.009)$. Higher operative mortality was associated with advanced patients $(\mathrm{P}<0.001)$, male sex $(\mathrm{P}<0.001)$, right-sided pneumonectomy $(\mathrm{P}<0.001)$, squamous cell carcinoma (SCC) $(\mathrm{P}=0.008)$, number of positive lymph nodes (npLNs) 5 or greater $(\mathrm{P}=0.010)$, and distant metastasis $(\mathrm{P}<0.001)$. However, induction radiotherapy $(\mathrm{RT})$ was a protective factor $(\mathrm{P}<0.001)$. The nomogram integrating all of the above independent predictors was well calibrated and had a relatively good discriminative ability, with a C-statistic of 0.687 and an area under the receiver operating characteristic (ROC) curve (AUC) of 0.682; moreover, DCA demonstrated that our model was clinically useful.

Conclusions: If pneumonectomy was considered inevitable, clinical decision-making based on this simple but efficient predictive nomogram could help minimize the risk of operative death and maximize the survival benefit.

Koywords: Pneumonectomy; lung cancer, operative mortality; nomogram

Submitted Apr 16, 2020. Accepted for publication Sep 19, 2020.

doi: $10.21037 /$ tlcr-20-561

View this article at: http://dx.doi.org/10.21037/tlcr-20-561 


\section{Introduction}

In 1933, Graham reported the first successful one-stage pneumonectomy for a gynecologist with lung squamous cell carcinoma (SCC) (1). Since then, pneumonectomy has become a standard surgical procedure in the treatment of lung neoplasms for a long period (2). However, with the development of lung-sparing resections (i.e., bronchoplastic and arterioplastic techniques, since 1946) and the advance of radiation therapies, targeted therapies and immunotherapies [i.e., stereotactic ablative radiotherapy (SABR), tyrosine kinase inhibitors (TKIs) and anti-programmed death (PD)1 antibody etc.] for locally advanced non-small cell lung cancer (LA-NSCLC), as well as the popularization of lowdose computed tomographic screening for earlier-stage lung cancer, the constituent ratio of pneumonectomy in the treatment of lung cancer has shown a significant declining trend over the past 20 years (3). The current guidelines recommend, in the premise of ensuring a negative margin, incorporating pneumonectomy into a multidisciplinary treatment strategy for patients with LA-NSCLC to obtain long-term survival (4). Data from the National Cancer Database demonstrated that the 5 -year overall survival (OS) rate of patients with pathologic stage IIIA (N2) NSCLC who underwent entire lung removal was up to $50.0 \%$ (5). In addition, several retrospective cohort studies comparing thoracoscopic pneumonectomy with open pneumonectomy showed the comparable perioperative outcomes and long-term survival $(6,7)$. Nevertheless, the higher pneumonectomy-related morbidity and mortality have undeniably influenced the intention to carry out this surgical procedure in clinical practice. Reported by a randomized controlled study, patients with stage IIIA (pN2) NSCLC who underwent induction chemoradiation followed by entire lung removal had an unacceptably high incidence of operative death (25.9\%) (8). Although a recent real-world study based on the French National Database reported that the operative mortality of pneumonectomy after neoadjuvant therapy was an acceptable rate of $5.7 \%$, the impression and apprehension of the high mortality remain (9). Therefore, to distinguish high-risk patients, it is necessary to identify the independent risk factors for postpneumonectomy death, i.e., clinical and pathological characteristics, induction therapy, and the extent of surgical resection etc., and to develop a model for precisely predicting this short-term outcome.

A nomogram, which is a simple and graphical predictive tool, can numerically calculate the risk probability of a clinical event tailored to individual patient (10-12). For many malignancies, the better predictive abilities of nomograms than the widely adopted tumor-node-metastasis (TNM) staging system have been demonstrated $(10,13)$. Recently, some studies have also explored the clinical applicability of nomograms for predicting short-term outcomes, including in-hospital mortality, postoperative pneumonia, perioperative complications, etc., which could help surgeons and nurses make appropriate clinical decisions and formulate postoperative management strategies for patients $(11,14,15)$. However, to date, a nomogram for precisely forecasting operative mortality in patients who underwent pneumonectomy remains unavailable.

Therefore, in this population-based study, the main purpose is to develop and validate the first predictive nomogram for numerically evaluating the individual risk probability of operative mortality for NSCLC patients after entire lung removal. And we present the following article in accordance with the Strengthening the Reporting of Observational Studies in Epidemiology (STROBE) reporting checklist (available at http://dx.doi.org/10.21037/ tlcr-20-561).

\section{Methods}

\section{Data source and cobort selection}

Patients in this retrospective longitudinal study were identified from the Surveillance, Epidemiology, and End Results (SEER) 18 registry custom database with an additional treatment fields (1975-2016 varying, released April 2019, www.seer.cancer.gov), which has a higher proportion of foreign-born persons $(17.9 \%)$ than the general United States population $(13.2 \%)$. SEER ${ }^{\star}$ Stat 8.3 .5 was installed to extract the information of patients who underwent pneumonectomy (surgical primary site codes: 40, 50-56, 65-66, 70) for lung cancer [the third edition of International Classification of Disease for Oncology (ICD-O-3): lung and bronchus, and behavior recode for analysis: malignant] from 1998 to 2016 $(\mathrm{N}=10,801)$. Four hundred and sixty-four patients with other primary malignant tumors incomplete follow-up times or outcomes, unavailable TNM staging, and age at diagnosis less than 18 years old were excluded from the final statistical analysis (Figure S1).

\section{Ethics statement}

The study was conducted in accordance with the Declaration 
of Helsinki (as revised in 2013). This retrospective study based on the SEER database was approved by the Surveillance Research Program in National Cancer Institute's Division of Cancer Control and Population Sciences (DCCPS) (reference number: 12101-Nov2018). Because of the retrospective nature of the research, the requirement for informed consent was waived.

\section{Covariates}

The continuous variable of age at diagnosis was divided into four age brackets $(\leq 40,41-60,61-70, \geq 70)$. Similarly, year of diagnosis was also transformed into a categorical variable and was grouped into 5-year intervals as follows: 1998-2002, 2003-2007, 2008-2012, and 2013-2016. The SEER summary $\mathrm{T}$ stage, $\mathrm{N}$ stage, and $\mathrm{M}$ stage were recategorized according to the eighth edition of the American Joint Committee on Cancer Staging Manual (16). Patients recorded as radiation prior to surgery, intraoperative radiation with or without other radiation before/after surgery, radiation before and after surgery, and sequence unknown but both were given were classified into the preoperative radiotherapy (RT) group. The histopathology based on the SEER variable "Histology ICD-O-3" was roughly categorized into adenocarcinoma (AC), SCC, adenosquamous cell carcinoma, neuroendocrine carcinoma (NC), carcinosarcoma, or other and unclassified carcinomas according to the 2015 World Health Organization (WHO) classification of tumors of the lung. In addition, these different measurement units were all converted to millimeters ( $\mathrm{mm}$ ).

\section{Outcomes}

Survival months were calculated from the date of pneumonectomy to the date of death or the last followup. This study defined operative mortality, which was the primary study outcome, as any death within 90 days (3 months) of pneumonectomy; accordingly, patients who did not die during the first 90 days after pneumonectomy were defined as 90-day survivors. All causes of death were regarded as uncensored cases in the OS analysis, but the cancer-specific survival (CSS) analysis included only death caused by lung cancer.

\section{Data analysis and statistical methods}

X-Tile version 3.6.1 software for Windows (Copyright Yale
University 2003, http://www.tissuearray.org/rimmlab/) was used to establish the optimal cut-off values for the number of positive lymph nodes (npLNs) $(\leq 4 / \geq 5)$ and the total number of tumors $(1 / \geq 2)$. Between patients presenting operative death and those who did not, the comparisons for frequencies and percentages were performed by chi-square tests or Fisher's exact test, and Student's $t$-test or the Mann-Whitney $\mathrm{U}$ test was applied to compare the continuous variables. These variables independently associated with operative mortality $(\mathrm{P}<0.05)$ were included in the multivariate logistic regression models. All above data analyses were performed by the Statistical Product and Service Solutions version 23.0 software (SPSS, IBM, Inc., Chicago, IL, USA, www.ibm.com/analytics/spss-statistics-software).

With the aid of $\mathrm{R}$ version 3.6.3 software for Windows (R Foundation for Statistical Computing, Vienna, Austria, www.r-project.org), the significant variables from the multivariate models were used to draw the graphical nomogram by using the "rms" package (15). Subsequently, a calibration curve was plotted to show the relationship between the nomogram-predicted probabilities and the observed probabilities. The $\mathrm{C}$-statistic and area under the receiver operating characteristic (ROC) curve (AUC) were used to measure the discrimination efficiency of this nomogram by bootstrap validation with 200 resamples from the original cohort, which was operated under the "pec" and "ROCR" packages, respectively. In addition, the novel decision curve analysis (DCA) was performed with the "rmda" package to calculate the net benefit of the forecast model by resampling 1,000 cases. The trend lines of the 5 -year CSS and OS rates, operative mortality, and the use of pneumonectomy from 1998 to 2016 and the Kaplan-Meier survival curves were graphed by using GraphPad Prism 7.0 software (San Diego, CA, USA, www.graphpad.com). The significance level for all tests was set at a $\mathrm{P}$ value less than 0.05 .

\section{Results}

\section{Baseline characteristics}

The baseline characteristics of this study cohort are summarized in Table 1. A total of 10,337 patients who underwent pneumonectomy (mean age: 62.5 years, range: 20-92 years) were retained in the final data analysis. The majority of patients were male (64.6\%), married (63.1\%) and uninsured $(60.0 \%)$. However, only $9.4 \%$ of patients were given induction RT or chemoradiotherapy (CRT) 
Table 1 Clinical and pathological variables for predicting operative mortality

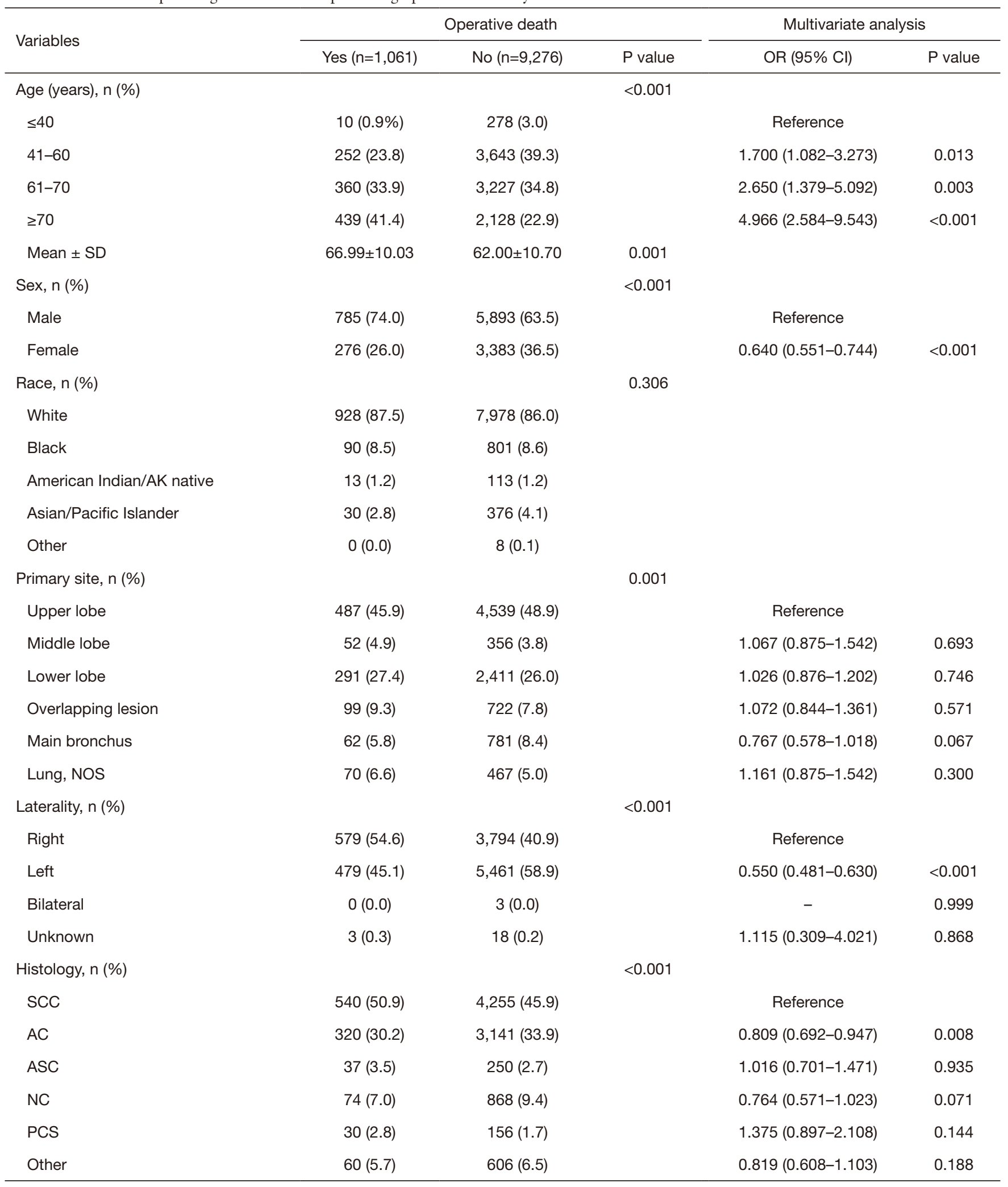

Table 1 (continued) 
Table 1 (continued)

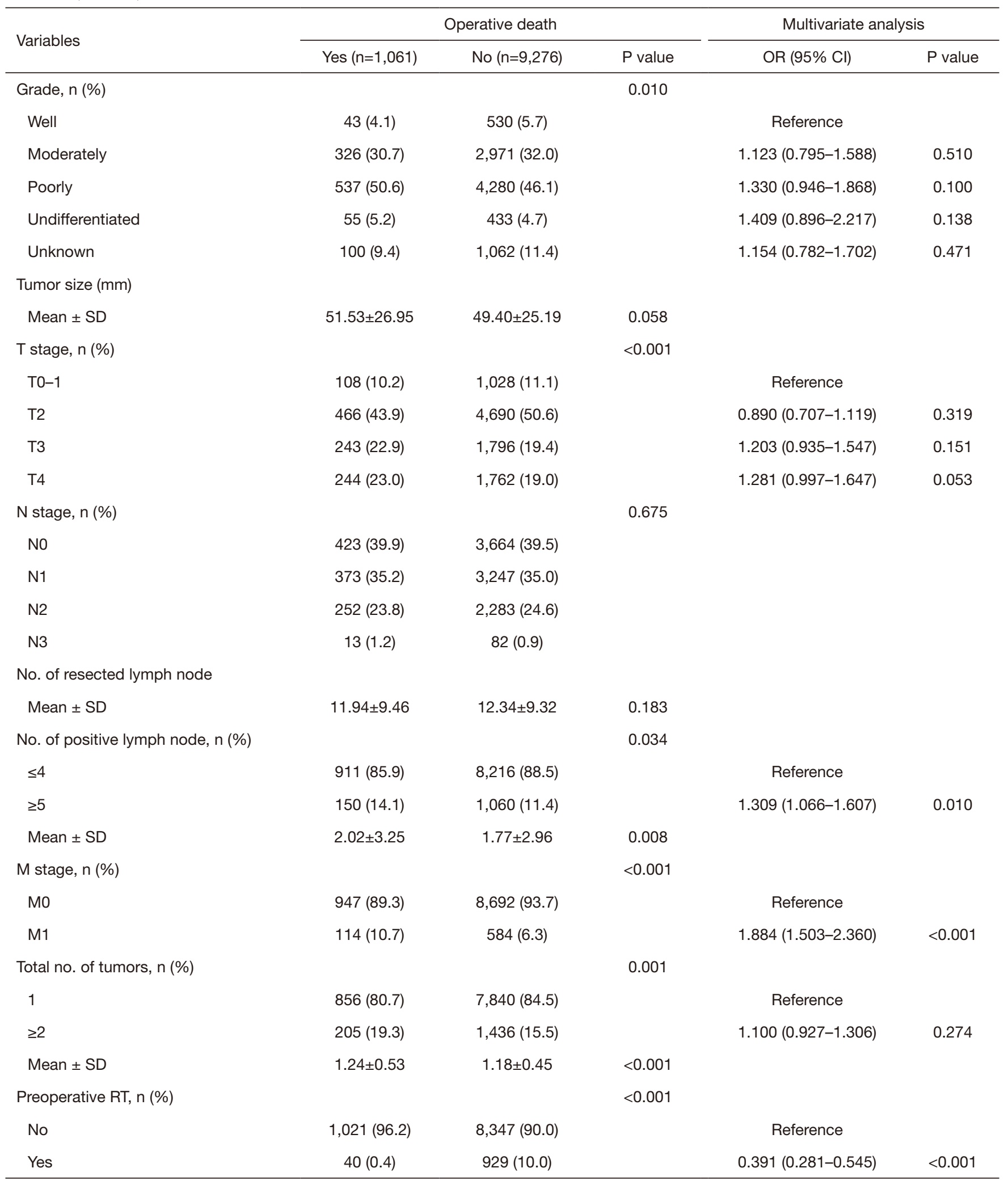

Table 1 (continued) 
Table 1 (continued)

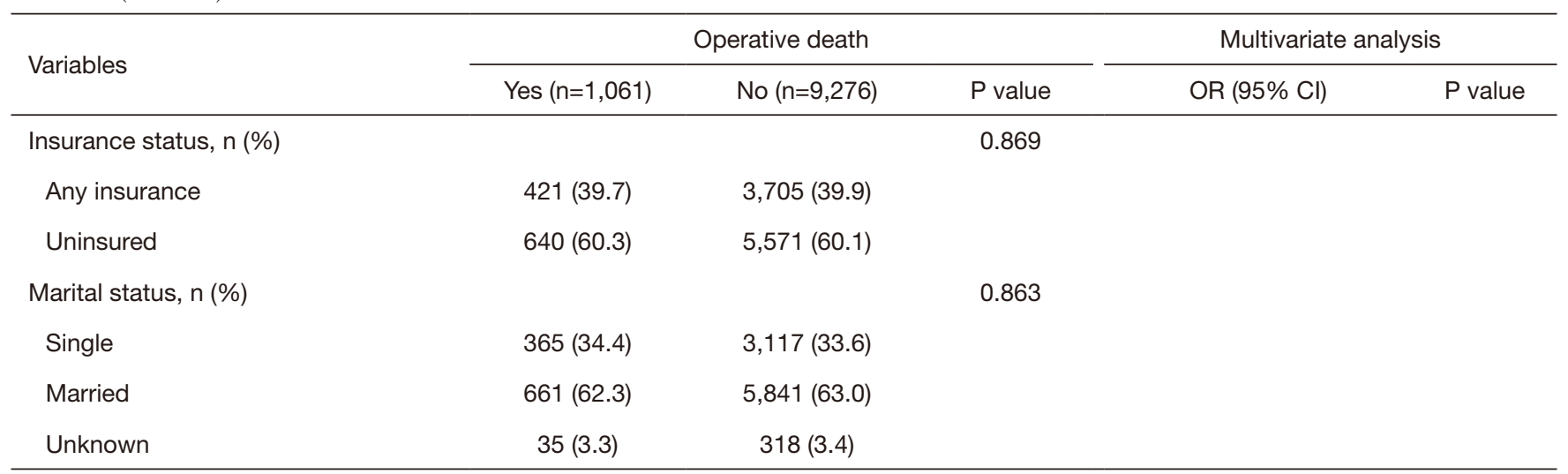

OR, odds ratio; $\mathrm{Cl}$, confidence interval; SD, standard deviation; NOS, not otherwise specified; SCC, squamous cell carcinoma; AC, adenocarcinoma; ASC, adenosquamous carcinoma; NC, neuroendocrine carcinoma; PCS, pulmonary carcinosarcoma; no., number; RT, radiotherapy.

before a pneumonectomy. The primary sites of the tumor were mostly located on the left side $(57.5 \%)$ and the upper lobe (48.6\%). The majority of pneumonectomies were due to locally advanced lung cancer (pathological stage II and III: $31.8 \%$ and $41.4 \%$, respectively), and 698 advanced patients underwent this surgical procedure. At postoperative pathological examination, SCC was the predominant histological subtype (46.4\%), followed by AC (33.5\%); in addition, the constituent ratios of histological grade I, II, III and IV were 5.5\%, 31.9\%, 46.6\% and 4.7\%, respectively.

\section{Operative mortality}

The overall operative mortality between 1998 and 2016 (almost 20 years) was $10.3 \%$, including a 30-day mortality of $4.2 \%$ ( 430 cases). The independent predictors identified by the multivariable logistic regression analysis for operative mortality are shown in Table 1. Compared to younger patients ( $\leq 40$ years), the odds ratio (OR) was $2.7[95 \%$ confidence interval (CI): 1.4-5.1; $\mathrm{P}=0.003$ ] for patients aged 61-70 years and 5.0 (95\% CI: 2.6-9.5; $\mathrm{P}<0.001)$ for patients aged $\geq 70$ years. Similarly, right-sided pneumonectomy was associated with approximately twice the operative mortality as left-sided pneumonectomy $(\mathrm{P}<0.001)$. In addition, male sex, SCC, npLNs 5 or greater and stage M1 were also indicated to be risk predictors for postoperative death. However, induction RT was a protective factor (OR: 0.4, 95\% CI: $0.3-0.5 ; \mathrm{P}<0.001)$.

\section{Nomogram for predicting operative mortality}

The above independent predictors identified by the multivariable analysis for operative mortality were integrated into the nomogram (Figure 1), which could be used to predict the risk probability of operative death for lung cancer patients who underwent pneumonectomy. The calibration curve for the probability of operative death in the bootstrap validation cohort demonstrated good accuracy between the actual values and prediction (Figure $2 A$ ). Furthermore, the numerical model showed a relatively good discriminative ability with a C-statistic of 0.687 and an AUC of 0.682 (Figure 2B). In addition, DCA and clinical impact curve analysis were introduced to further evaluate the clinical utility of this graphical model. The curves (Figure 3) indicated that if the threshold probability was in the range of $0.3-0.7$, using this graphical model to predict operative death added more net benefit.

\section{Trend of pneumonectomy and long-term survival}

Data from the SEER database demonstrated a significant decrease in the constitute ratio of pneumonectomy in the surgical treatment of lung cancer over the past two decades [1998-2002 (cohort 1): 6.7\%; 2003-2007 (cohort 2): 5.9\%; 2008-2012 (cohort 3): 5.1\%; 2013-2016 (cohort 4): 2.9\%; $\mathrm{P}=0.001]$. Similarly, there was a significant declining trend in the postpneumonectomy death rate $(10.8 \%, 9.7 \%$, $11.8 \%$, and $9.1 \%$ in cohorts $1,2,3$, and 4 , respectively; 


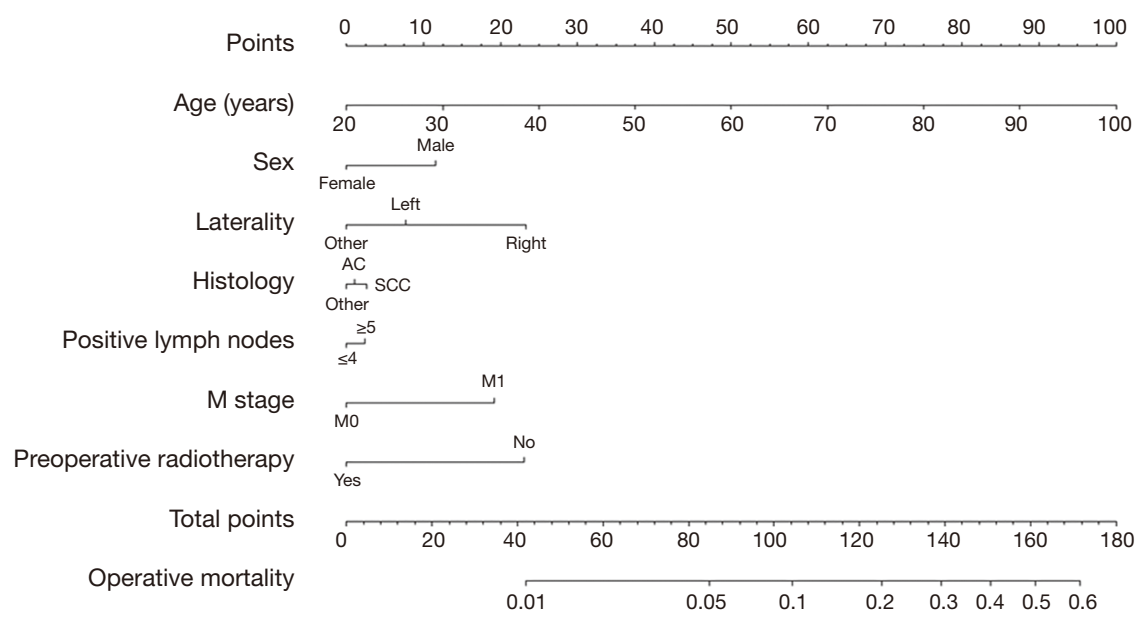

Figure 1 Nomogram for predicting the operative mortality of patients who underwent pneumonectomy for lung cancer. SCC, squamous cell carcinoma.
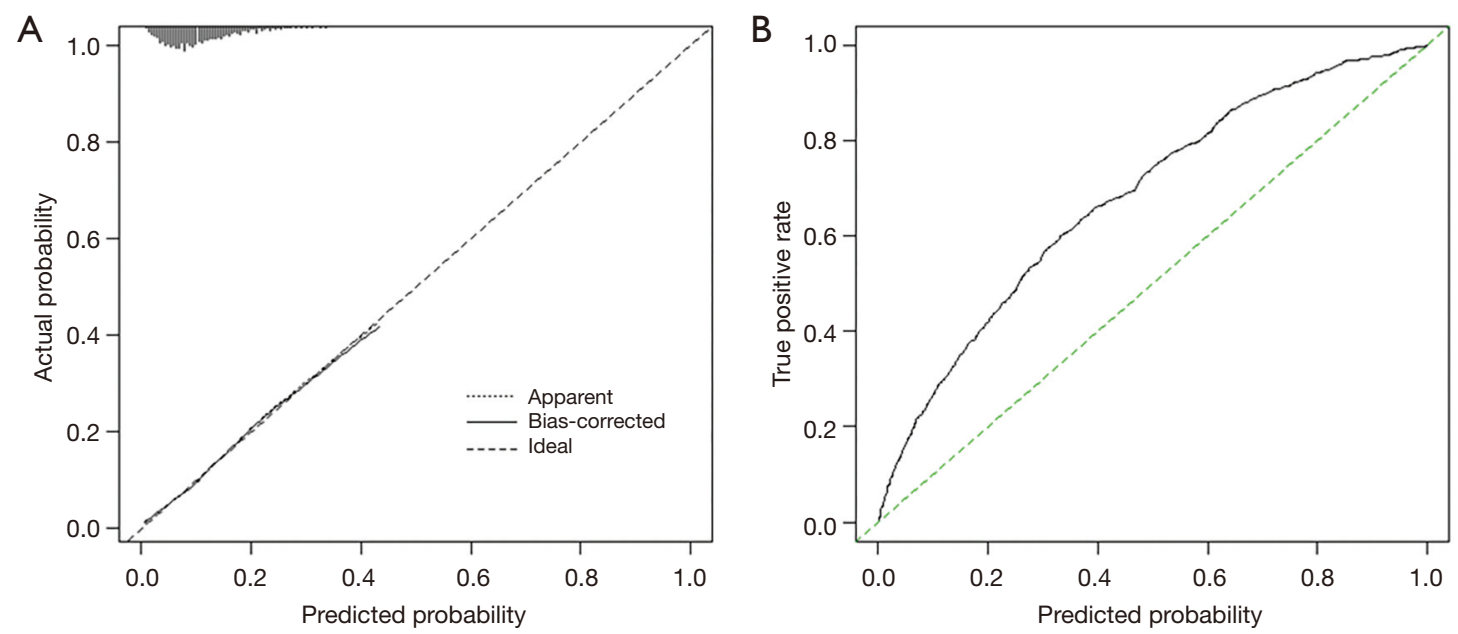

Figure 2 The calibration curve (A) and ROC curve (B) according to the nomogram for predicting the operative mortality of patients who underwent pneumonectomy for lung cancer in the bootstrap validation. ROC, receiver operating characteristic.

\section{$\mathrm{P}=0.009 ;$ Figure $4 A$ ).}

The estimated 5-year OS and CSS rates in operative survivors were $40.2 \%$ and $55.5 \%$, respectively, and there were statistically significant increases in the 5 -year OS rate from $35.8 \%$ in 1998 to $48.1 \%$ in $2012(\mathrm{P}<0.001)$ and in the 5 -year CSS rate from $49.9 \%$ in 1998 to $62.7 \%$ in $2012(\mathrm{P}<0.001$, Figure $4 A)$. Moreover, the running log-rank statistics showed that the widely adopted TNM staging system was still valid in separating the long-term survival of pathological stage I-IV lung cancers after pneumonectomy (all $\mathrm{P}<0.001$, Figure 4B,C).

\section{Discussion}

In this population-based study, age, sex, laterality, histology, positive lymph nodes, metastasis, and preoperative RT were identified by multivariate logistic regression analysis as independent risk factors for predicting postpneumonectomy death. Subsequently, the above seven predictors were incorporated into a nomogram to numerically calculate the 

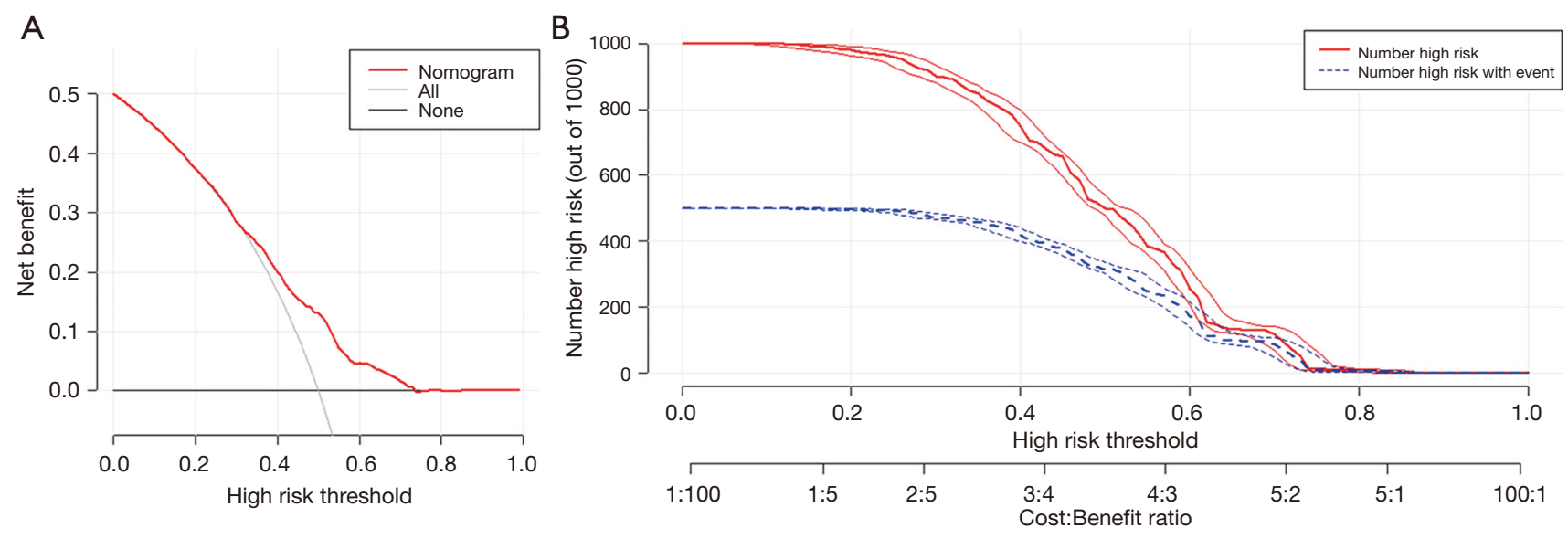

Figure 3 DCA (A) and clinical impact curve (B) to calculate the net benefit of the nomogram for predicting the operative mortality of patients who underwent pneumonectomy for lung cancer in bootstrap validation. DCA, decision curve analysis.

risk probability of operative death tailored to individual patients.

In the National Surgical Quality Improvement Program (NSQIP) database, surgical mortality was defined as any cause of death occurring within 30 days after noncardiac thoracic surgery. The 30-day mortality could not only assess the index operative risk but also weigh the purported survival benefit and the unintended postoperative death $(17,18)$. Generally, for patients who underwent routine thoracic surgery, such as sublobar resection and lobectomy, the 30-day interval was close enough to capture all operation-related deaths (19). However, recent studies on pneumonectomy have reported that 90 -day mortality $(3.0-21.0 \%)$ is approximately twice as high as 30 -day mortality $(1.5-12.0 \%)(7,18,20-24)$. In other words, the operative risk of entire lung removal did not end at 30 days or discharge. Therefore, to reduce operative mortality and successfully manage postoperative complications, intensive care, treatment, and follow-up lasting for 90 days or more may be more appropriate for patients who undergo pneumonectomy.

Similar to the findings of a host of previous studies, our multivariate regression analysis also found that the risk of operative mortality increased with male sex, advanced age, right-sided pneumonectomy, and SCC $(9,23,25)$. In addition, the npLNs $(\geq 5)$ was first identified as a risk factor for postpneumonectomy death, but the traditional node staging did not show this predictive value; similarly, distant metastasis was also associated with higher operative mortality, which cautioned us that the advanced stage was a poor indication not only for long-term survival but also for short-term outcomes. Therefore, if pneumonectomy was considered inevitable before surgery, accurate staging techniques, such as positron emission tomography, endobronchial ultrasonography, or endoscopic ultrasonography etc., may be necessary.

However, the risk of operative mortality after induction RT and pneumonectomy remains controversial. A phase III study, administered by the Radiation Therapy Oncology Group (RTOG9309) evaluated the survival benefit of surgical resection after CRT for stage IIIA (N2) NSCLC. Of 54 patients who underwent pneumonectomy, 14 patients (25.9\%) experienced operative death within 30 days (8). Similarly, in two other randomized controlled trials, the 30 -day mortality of LA-NSCLC patients after induction CRT and pneumonectomy was $9.6 \%(10 / 104)$ and $6.9 \%$ $(5 / 72)$, respectively $(26,27)$. The findings of recently reported big data study based on the Society of Thoracic Surgery General Thoracic Surgery Database (STS GTDB) were in line with this trend (28). Therefore, complete lung removal may not be suitable for LA-NSCLC patients after CRT unless operative death can be avoided. However, as reported by a single center prospective study, the mortality after pneumonectomy between patients receiving and not receiving induction $\mathrm{CT}$ (cisplatin-based regimen, two cycles) and RT (50.4 Gy) for LA-NSCLC showed no significant difference (29). Moreover, a study reported that even though patients received concurrent induction CT and full-dose RT (59.4-66.6 Gy), the operative mortality of pneumonectomy was nil (30). More interestingly, the French National General Thoracic Surgery Database (EPITHOR) reported that, adjusted for propensity scores, neoadjuvant CRT or RT was significantly associated with a 


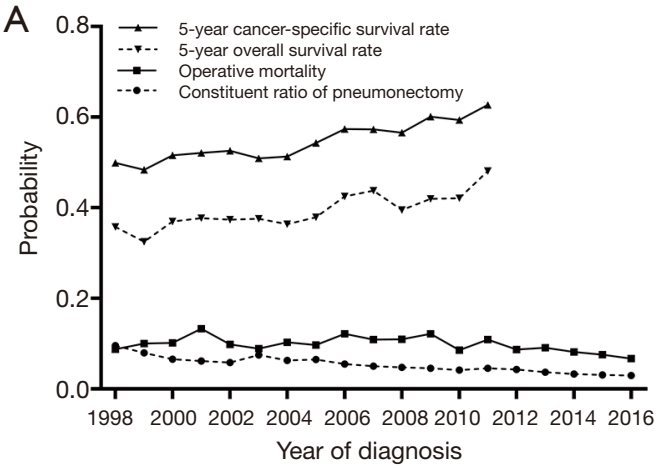

B
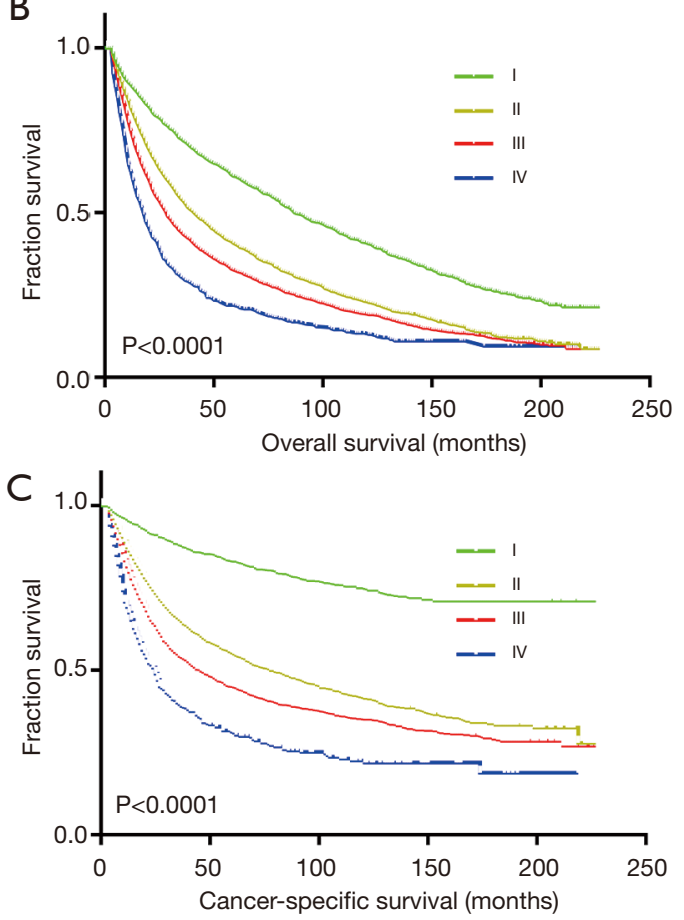

Figure 4 Trends of the use of pneumonectomy, and postpneumonectomy survival rates. (A) Changes in the 5-year CSS rates, the 5-year OS rates, the postpneumonectomy mortality, and the constituent ratios of pneumonectomy in the surgical treatment of lung cancer; (B) the OS curves of 90-day survivors after pneumonectomy according to the combined TNM staging; $(\mathrm{C})$ the CSS curves of 90-day survivors after pneumonectomy according to the combined TNM staging. CSS, cancer-specific survival; OS, overall survival; TNM, tumor-node-metastasis.

decreased risk of operative mortality, which was consistent with the findings of our population-based study (9). On the basis of the available evidence from the literature, we presumed that the following limitations may lead to this striking finding.
First, the time spans of this retrospective study covered almost 20 years; therefore, selection bias was inevitable. Second, the proportions of the use of induction RT or CRT in our SEER database cohort and the French EPITHOR cohort were lower than those of the American STS GTDB cohort (9.4\% and $2.7 \%$ vs. $16.7 \%$ ), which may result in treatment bias $(9,28)$. Third, the incidences of postpneumonectomy complications were not documented in the SEER database, which could have result in a severe short-term prognosis $(7,9)$. In addition, preoperative patient selection, intraoperative preventive actions, and postoperative intensive care, especially for patients who received induction therapy, to avoid the occurrence of these dreaded complications after whole lung removal could not be retrieved from the database. However, these processes, including performance status evaluation, pulmonary function tests, right heart function tests, bronchial stump coverage, postoperative volume of fluids in the first 12 hours, postoperative pulmonary artery pressure measurements, chest tube handling, etc., have been reported to significantly reduce the incidence of postpneumonectomy morbidity and mortality $(31,32)$. Fourth, the hospital volumes of surgical procedures and radiotherapies had significant effect on postpneumonectomy mortality and radiation toxicity to the remaining lung, but this factor was not considered in our study $(33,34)$. Undeniably, the extensive surgery, airway management, RT and nursing experience available in the United States may have a positive contribution to this favorable finding. The above descriptions were also the limitations of this population-based study.

To visualize and integrate these independent risk factors, a graphical nomogram was built to generate intuitive and numerical risk probabilities of postpneumonectomy death for individual patients. Moreover, this predictive model showed a relatively good discriminative ability and clinical net benefit in bootstrap invalidation, and all the factors listed could be easily obtained from clinical and pathological data, which facilitates the clinical applicability during postoperative decision making for pneumonectomy. However, of note, bootstrapping was selected as the validation method in the present study, and further external validation should be performed in clinical practice.

\section{Conclusions}

Although the high overall mortality of entire lung removal between 1998 and 2016 remained in the SEER database, a significant declining trend was also observed. Moreover, 
patients underwent pneumonectomy for lung cancer and did not experience postoperative death could obtain the chance for long-term survival. Therefore, in the present study, the graphical nomogram with a relatively good performance could weigh the risk of operative death and survival benefit after pneumonectomy.

\section{Acknowledgments}

Presented at the 28th Annual Meeting of The European Society for Thoracic Surgeon, The Hague, The Netherlands, 31 May-3 June, 2020.

Funding: The preparation, English editing and publication of this manuscript were supported by the National Key R\&D Plan (No. 2017YFC1308700), the Ministry of Science and Technology of the People's Republic of China.

\section{Footnote}

Reporting Checklist: The authors have completed the STROBE reporting checklist. Available at http://dx.doi. org/10.21037/tlcr-20-561

Conflicts of Interest: All authors have completed the ICMJE uniform disclosure form (available at http://dx.doi. org/10.21037/tlcr-20-561). The authors have no conflicts of interest to declare.

Ethical Statement: The authors are accountable for all aspects of the work in ensuring that questions related to the accuracy or integrity of any part of the work are appropriately investigated and resolved. The study was conducted in accordance with the Declaration of Helsinki (as revised in 2013). This retrospective study based on the SEER database was approved by the Surveillance Research Program in National Cancer Institute's Division of Cancer Control and Population Sciences (DCCPS) (reference number: 12101-Nov2018). Because of the retrospective nature of the research, the requirement for informed consent was waived.

Open Access Statement: This is an Open Access article distributed in accordance with the Creative Commons Attribution-NonCommercial-NoDerivs 4.0 International License (CC BY-NC-ND 4.0), which permits the noncommercial replication and distribution of the article with the strict proviso that no changes or edits are made and the original work is properly cited (including links to both the formal publication through the relevant DOI and the license). See: https://creativecommons.org/licenses/by-nc-nd/4.0/.

\section{References}

1. Graham EA, Singer JJ. Successful removal of an entire lung for carcinoma of the bronchus. CA Cancer J Clin 1974;24:238-42.

2. Fell SC. Special article: a brief history of pneumonectomy. 1999. Chest Surg Clin N Am 2002;12:541-63.

3. Hancock J, Rosen J, Moreno A, et al. Management of clinical stage IIIA primary lung cancers in the National Cancer Database. Ann Thorac Surg 2014;98:424-32; discussion 432.

4. Postmus PE, Kerr KM, Oudkerk M, et al. Early and locally advanced non-small-cell lung cancer (NSCLC): ESMO Clinical Practice Guidelines for diagnosis, treatment and follow-up. Ann Oncol 2017;28:iv1-21.

5. Herskovic A, Mauer E, Christos P, et al. Role of postoperative radiotherapy in pathologic stage IIIA (N2) non-small cell lung cancer in a prospective nationwide oncology outcomes database. J Thorac Oncol 2017;12:302-13.

6. Yang CJ, Yendamuri S, Mayne NR, et al. The role of thoracoscopic pneumonectomy in the management of non-small cell lung cancer: a multicenter study. J Thorac Cardiovasc Surg 2019;158:252-64.e2.

7. Blanc K, Zaimi R, Dechartres A, et al. Early acute respiratory distress syndrome after pneumonectomy: presentation, management, and short- and long-term outcomes. J Thorac Cardiovasc Surg 2018;156:1706-14.e5.

8. Albain KS, Swann RS, Rusch VW, et al. Radiotherapy plus chemotherapy with or without surgical resection for stage III non-small-cell lung cancer: a phase III randomised controlled trial. Lancet 2009;374:379-86.

9. Thomas PA, Berbis J, Baste JM, et al. Pneumonectomy for lung cancer: contemporary national early morbidity and mortality outcomes. J Thorac Cardiovasc Surg 2015;149:73-82.

10. Cheng B, Wang C, Zou B, et al. A nomogram to predict outcomes of lung cancer patients after pneumonectomy based on 47 indicators. Cancer Med 2020;9:1430-40.

11. Sakamoto Y, Yamauchi Y, Yasunaga H, et al. Development of a nomogram for predicting in-hospital mortality of patients with exacerbation of chronic obstructive pulmonary disease. Int J Chron Obstruct Pulmon Dis 2017;12:1605-11.

12. Iasonos A, Schrag D, Raj GV, et al. How to build and 
interpret a nomogram for cancer prognosis. J Clin Oncol 2008;26:1364-70.

13. Zheng XQ, Huang JF, Lin JL, et al. Incidence, prognostic factors, and a nomogram of lung cancer with bone metastasis at initial diagnosis: a population-based study. Transl Lung Cancer Res 2019;8:367-79.

14. Zhang D, Zhuo H, Yang G, et al. Postoperative pneumonia after craniotomy: incidence, risk factors and prediction with a nomogram. J Hosp Infect 2020;105:167-75.

15. Zhou ZR, Wang WW, Li Y, et al. In-depth mining of clinical data: the construction of clinical prediction model with R. Ann Transl Med 2019;7:796.

16. Rami-Porta R, Bolejack V, Crowley J, et al. The IASLC Lung Cancer Staging Project: Proposals for the Revisions of the T Descriptors in the Forthcoming Eighth Edition of the TNM Classification for Lung Cancer. J Thorac Oncol 2015;10:990-1003.

17. Khuri SF, Daley J, Henderson W, et al. The Department of Veterans Affairs' NSQIP: the first national, validated, outcome-based, risk-adjusted, and peer-controlled program for the measurement and enhancement of the quality of surgical care. National VA Surgical Quality Improvement Program. Ann Surg 1998;228:491-507.

18. McMillan RR, Berger A, Sima CS, et al. Thirty-day mortality underestimates the risk of early death after major resections for thoracic malignancies. Ann Thorac Surg 2014;98:1769-74; discussion 1774-5.

19. Dickhoff C, Dahele M, de Langen AJ, et al. Populationbased patterns of surgical care for stage IIIA NSCLC in the Netherlands between 2010 and 2013. J Thorac Oncol 2016;11:566-72.

20. Mansour Z, Kochetkova EA, Ducrocq X, et al. Induction chemotherapy does not increase the operative risk of pneumonectomy! Eur J Cardiothorac Surg 2007;31:181-5.

21. Thibout Y, Guibert B, Bossard N, et al. Is pneumonectomy after induction chemotherapy for non-small cell lung cancer a reasonable procedure? A multicenter retrospective study of 228 cases. J Thorac Oncol 2009;4:1496-503.

22. Tabutin M, Couraud S, Guibert B, et al. Completion pneumonectomy in patients with cancer: postoperative survival and mortality factors. J Thorac Oncol 2012;7:1556-62.

23. Weder W, Collaud S, Eberhardt WEE, et al. Pneumonectomy is a valuable treatment option after neoadjuvant therapy for stage III non-small-cell lung cancer. J Thorac Cardiovasc Surg 2010;139:1424-30.

24. Doddoli C, Barlesi F, Trousse D, et al. One hundred consecutive pneumonectomies after induction therapy for non-small cell lung cancer: an uncertain balance between risks and benefits. J Thorac Cardiovasc Surg 2005;130:416-25.

25. Safi S, Benner A, Walloschek J, et al. Development and validation of a risk score for predicting death after pneumonectomy. PLoS One 2015;10:e0121295.

26. Thomas M, Rübe C, Hoffknecht P, et al. Effect of preoperative chemoradiation in addition to preoperative chemotherapy: a randomised trial in stage III non-smallcell lung cancer. Lancet Oncol 2008;9:636-48.

27. van Meerbeeck JP, Kramer GWPM, Van Schil PEY, et al. Randomized controlled trial of resection versus radiotherapy after induction chemotherapy in stage IIIA-N2 non-small-cell lung cancer. J Natl Cancer Inst 2007;99:442-50.

28. Shapiro M, Swanson SJ, Wright CD, et al. Predictors of major morbidity and mortality after pneumonectomy utilizing the Society for Thoracic Surgeons General Thoracic Surgery Database. Ann Thorac Surg 2010;90:927-34; discussion 934-5.

29. Ng T, Birnbaum AE, Fontaine JP, et al. Pneumonectomy after neoadjuvant chemotherapy and radiation for advanced-stage lung cancer. Ann Surg Oncol 2010;17:476-82.

30. Sonett JR, Suntharalingam M, Edelman MJ, et al. Pulmonary resection after curative intent radiotherapy ( $>59$ Gy) and concurrent chemotherapy in non-small-cell lung cancer. Ann Thorac Surg 2004;78:1200-5; discussion 1206.

31. Swartz DE, Lachapelle K, Sampalis J, et al. Perioperative mortality after pneumonectomy: analysis of risk factors and review of the literature. Can J Surg 1997;40:437-44.

32. Kesler KA. Can we make pneumonectomy great again? J Thorac Cardiovasc Surg 2018;156:1704-5.

33. Birkmeyer JD, Siewers AE, Finlayson EVA, et al. Hospital volume and surgical mortality in the United States. N Engl J Med 2002;346:1128-37.

34. Wang W, Men Y, Wang J, et al. Postoperative radiotherapy is effective in improving survival of patients with stage pIII-N2 non-small-cell lung cancer after pneumonectomy. BMC Cancer 2019;19:478.

Cite this article as: Yu X, Gao S, Xue Q, Tan F, Gao Y, Mao Y, Wang D, Zhao J, Li Y, Wang F, Cheng H, Zhao C, Mu J. Development of a nomogram for predicting the operative mortality of patients who underwent pneumonectomy for lung cancer: a population-based analysis. Transl Lung Cancer Res 2021;10(1):381-391. doi: 10.21037/tlcr-20-561 
Supplementary

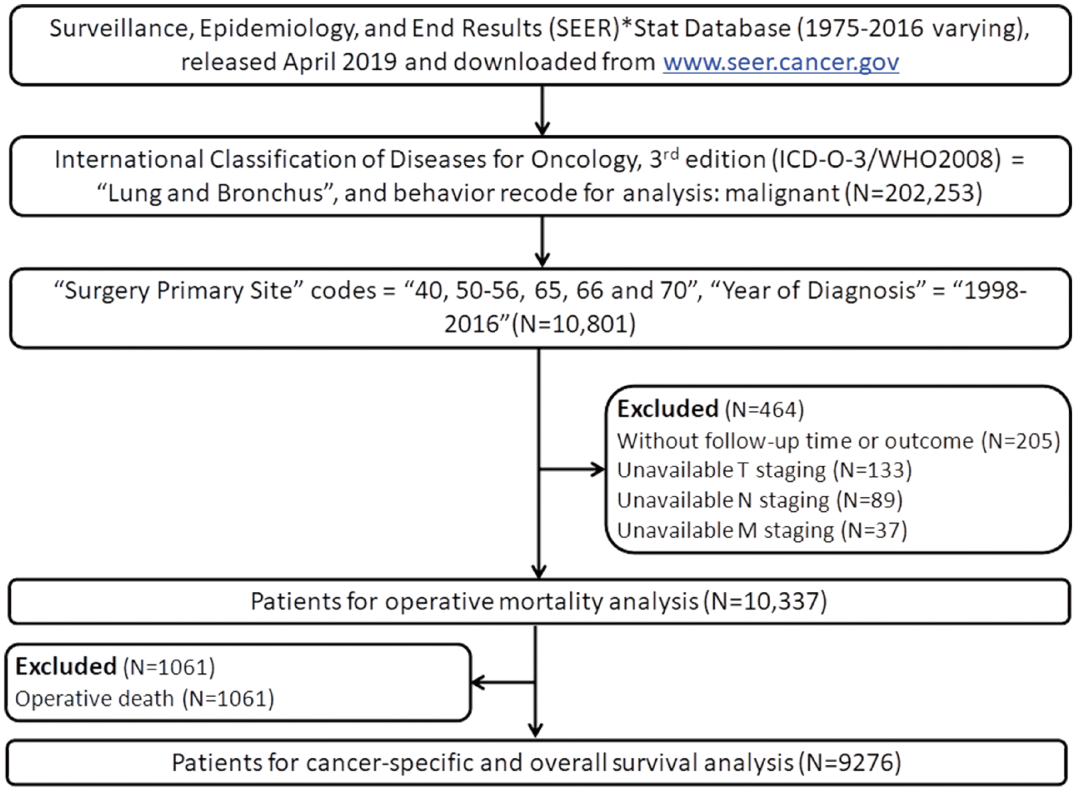

Figure S1 Patient selection algorithm. 